\title{
VISAT: DIFICULDADES DA EQUIPE MULTIPROFISSIONAL DE UM CEREST DE SC
}

\author{
VISAT: DIFFICULTIES OF THE MULTIPROFESSIONAL TEAM OF A SC CEREST
}

Isabel Domingos Ceron ${ }^{1}$ Maristella Siqueira Pra²

Recebido em: 04 jul. 2017

Aceito em: 05 maio 2018

RESUMO: Estudo com objetivo de identificar as dificuldades encontradas pela equipe multiprofissional de um Centro de Referência em Saúde do Trabalhador (CEREST) Macrorregional de Santa Catarina, quanto a realização de ações de vigilância em saúde do trabalhador. Pesquisa de abordagem qualitativa, descritiva, exploratória e de campo. $\mathrm{O}$ estudo foi desenvolvido com os profissionais que compõe a equipe do referido CEREST, no período de fevereiro a maio 2017. Para coleta de dados foi realizada uma entrevista com roteiro semi-estruturado com o total dos profissionais da equipe que atualmente são quatro. A análise dos dados foi realizada a partir da análise de conteúdo de Minayo a qual preconiza a categorização dos dados. As principais dificuldades apontadas pela equipe estão vinculadas a falta de recursos humanos, a área de abrangência do CEREST, a diversidade da área de atuação das empresas, a localização das empresas a serem fiscalizadas estarem muito distantes umas das outras em locais de difícil acesso e a falta de identificação destas empresas. Os fatores relacionados a falta de recursos humanos, interferem diretamente no processo de trabalho da equipe, que atualmente tem suas atividades voltadas a fiscalização dos ambientes de trabalho, de casos com datas préestabelecidas pelo MP. A equipe optou em organizar suas ações em dois núcleos; capacitações e fiscalizações.

Palavras-chave: Trabalho. Saúde do Trabalhador. Vigilância em Saúde.

ABSTRACT: This study aimed to identify the difficulties encountered by the multiprofessional team of a Reference Center for Occupational Health (CEREST) Macroregional of Santa Catarina, regarding the Health surveillance. Research of qualitative, descriptive, exploratory and field approach. The study was developed with the professionals that make up the CEREST team from February to May 2017. For data collection, an interview with a semi-structured script was carried out with the total of the professionals of the team, who are currently four. The analysis of the data was carried out from the analysis of Minayo which advocates the categorization of data. The main difficulties The lack of human resources, the scope of CEREST, the diversity of the area of activity of the companies, the location of the companies to be inspected are far Of the others in places of difficult access and the lack of identification of these companies. The factors related to the lack of human resources directly interfere in the work process of the team, which currently has its activities focused on the supervision of work environments, of cases with dates established by the MP. The team chose to organize their actions in two nuclei; Training and supervision.

Keywords: Work. Worker's Health. Health Surveillance.

\footnotetext{
1 Universidade do Extremo Sul Catarinense.

2 Universidade do Extremo Sul Catarinense.
} 


\section{CONTEXTUALIZAÇÃO DO TEMA}

Trabalhar se constitui numa parte importante da vida do ser humano, e a importância deste na vida das pessoas vai muito além de que, através dele, satisfazemos nossas necessidades básicas; tem a ver também com realização pessoal, com sentir-se útil e encontrar sentido para os dias (KANAANE, 1999).

O trabalho, por si só, é revelador da nossa humanidade, uma vez que possibilita ação transformadora sobre a natureza e si mesmo. Além disso, a nossa capacidade inventiva e criadora é exteriorizada através do ofício que realizamos. Todas essas mudanças remeteram a novos conceitos de trabalho e relações no trabalho, observandose aqui um maior adoecimento do trabalhador e por este motivo surge então a necessidade de maior discussão do assunto saúde do trabalhador.

Refletir sobre o processo saúde e doença requer pensar nas questões relacionadas ao trabalho e suas repercussões na vida do trabalhador e de sua família. De acordo com vários estudos, entende-se que são diversas as causas e fatores que interferem no aparecimento de doenças relacionadas ao trabalho.

Atualmente, as novas tecnologias e formas de organização que vêm sendo implantadas nos processos de trabalho para produções mais dinâmicas, tem influenciado diretamente sobre a saúde do trabalhador (TORRES et al., 2011). A Saúde do Trabalhador, é uma área de pesquisa consolidada como produção de conhecimentos específico em saúde coletiva, sendo também, um tema de estudo transversal associando conhecimentos de epidemiologia, ciências sociais e planejamento e estudos de gêneros, doenças e saúde ambiental. (STRAUSZ, 2014).

A Saúde do Trabalhador refere-se a um campo do saber que visa compreender as relações entre o trabalho e o processo saúde/doença (LACAZ; 1996).

A vigilância em saúde do trabalhador é uma ação típica de saúde pública e, portanto, vinculada diretamente ao Sistema Único de Saúde (SUS). É a partir do SUS que se pode falar em VISAT.

O campo da Saúde do Trabalhador no Brasil sofreu influência direta dos movimentos sindicais e suas reivindicações por melhores condições de trabalho e de saúde.

"Com a regulamentação da Lei o 8.080/90, ficou definido que cabe ao Sistema Único de Saúde (SUS) atuar na assistência, na vigilância e no controle de agravos à saúde relacionados ao trabalho" (QUILIÃO; FASSA; RESTREPO, 2013, p.258).

O SUS, é um sistema que articula políticas e outras estruturas do Estado brasileiro. É necessário então, pensar uma vigilância voltada para a saúde no trabalho vinculando a rede de saúde, para dar conta de seu objetivo maior, ou seja, vigiar e intervir nos processos e ambientes de trabalho onde existam fatores determinantes de perda da saúde da população que trabalha. 
Logo, são os Centros de Referência em Saúde do Trabalhador (CEREST's) que devem desencadear a VISAT, seja disseminando-a na própria rede SUS, onde ela deve permear grande parte das ações.

A partir dessas reflexões surgiu o problema de pesquisa com o tem: Quais as dificuldades de vigilância em saúde do trabalhador encontradas pela equipe multiprofissional de um Centro de Referência em Saúde do Trabalhador (CEREST) de Santa Catarina?

Buscando identificar as dificuldades encontradas pela equipe multiprofissional surgiu as seguintes hipóteses:

Equipe multiprofissional defasada em relação a área de abrangência;

Falta de treinamento e capacitação da equipe;

Falta de recursos materiais e investimento para desenvolver ações em saúde do trabalhador.

Diante das reflexões sobre a temática, tem-se como objetivo geral identificar as dificuldades de vigilância em saúde do trabalhador encontradas pela equipe multiprofissional de um CEREST de Santa Catarina. Além do objetivo geral para elencarse, surge os objetivos específicos: Conhecer as ações de vigilância em saúde do trabalhador desenvolvidas pelo CEREST; identificar a forma como as ações de vigilância em saúde do trabalhador são realizadas pelo CEREST; descrever as ações de vigilância em saúde do trabalhador desenvolvidas pelo CEREST.

\section{MÉTODOS}

Pesquisa de abordagem qualitativa, descritiva, exploratória do campo. O estudo foi realizado em um Centro de Referência em Saúde do Trabalhador (CEREST) de uma região de Santa Catarina.

A análise de dados foi iniciada após a aprovação do Comitê de Ética em Pesquisa da instituição UNESC.

O estudo foi realizado com os profissionais que compõe atualmente a equipe multiprofissional do CEREST, sendo hoje quatro (04) profissionais.

O questionário semiestruturado se deu como roteiro flexível visando obter respostas aos objetivos da pesquisa.

A análise dos dados foi realizada a partir da análise de conteúdo de Minayo, a qual preconiza a categorização dos dados; que visa à interpretação de material de caráter qualitativo, assegurando uma descrição objetiva, sistemática e com a riqueza detalhes no momento da coleta dos dados.

Para preservar o sigilo decorrente das entrevistas realizadas, de acordo com as diretrizes e normas regulamentadoras da Res. 510/16 (CNS) que envolvem pesquisa com 
Seres Humanos e Grupos Vulneráveis, utilizou-se a letra "E” para os profissionais da equipe multidisciplinar, seguida do respectivo número - E1 a E14.

A coleta de dados foi iniciada após a aprovação da pesquisa pelo Comitê de Ética em Pesquisa da UNESC; pelo parecer 1.870.468.

\section{RESULTADOS E DISCUSSÃO}

\section{PERFIL DOS ENTREVISTADOS:}

Quanto ao gênero todos são do sexo feminino. Quanto a idade variou de 44 anos a 54 anos; Quanto ao tempo de atuação no serviço variou de 4 anos a 8 meses. Das entrevistadas 3 possuem curso superior e 1 das entrevistadas nível técnico. Participaram de capacitações relacionado as fiscalizações: 3 das entrevistadas e somente 1 das entrevistadas atua na sua área de formação. As atividades desempenhadas exigiram apenas um tempo mínimo de 2 semanas de capacitação em VISAT.

\section{AÇÕES DESENVOLVIDAS PELO CEREST}

Segundo a equipe multidisciplinar as ações desenvolvidas pelo CEREST estão relacionadas à fiscalização do ambiente de trabalho; promoção, prevenção, assistência e vigilância em saúde do trabalhador; além de capacitações da equipe.

\begin{tabular}{l|l} 
Quadro no 2 - Ações desenvolvidas pelo CEREST \\
\hline Ações desenvolvidas pelo CEREST & Entrevistado (E1 a E4) \\
\hline Fiscalização dos ambientes de trabalho & 03 (E1, E2 e E3) \\
\hline Promoção à saúde do trabalhador & 02 (E1 e E4) \\
\hline Prevenção em saúde do trabalhador & $01(\mathrm{E} 4)$ \\
\hline Assistência à saúde do trabalhador & $01(\mathrm{E} 4)$ \\
\hline Vigilância em saúde do trabalhador & $01(\mathrm{E} 4)$ \\
\hline Capacitações & $01(\mathrm{E} 1)$ \\
\hline
\end{tabular}

Nesta categoria, a fiscalização do processo de trabalho foi a ação mais citada pelos entrevistados, sendo uma das atribuições do CEREST.

A fiscalização do processo de trabalho é uma das atribuições do Centro de Referência em Saúde do Trabalhador - CEREST, segundo os profissionais E1, E2 e E3:

E1 - "Fiscalização dos ambientes e processos de trabalho[...]"

E 2 e 3 - "Fiscalização dos ambientes de trabalho de empresas e/ou órgãos com CNPJ; fiscalizações voltadas a saúde do trabalhador".

Rosa et al. (2016) e Sanchez (2009), referem que as ações de fiscalização dos ambientes de trabalho consistem de fato, em umas das atividades a ser desenvolvida pelo 
CEREST, sendo possível a partir desta ação, avaliar as condições de saúde dos trabalhadores fazendo um diagnóstico dos ambientes de trabalho.

Quando da criação da RENAST e implementação dos CERESTs; a Vigilância em Saúde do Trabalhador passou a contar com diversas atribuições desenvolvidas por esses centros, dentre essas as fiscalizações dos ambientes de trabalho. (NEIVERTH et al.,2016).

Segundo os entrevistados, o CEREST também desenvolve ações de promoção, prevenção, assistência e vigilância em saúde do trabalhador da rede de saúde.

E1 - "[...]promovendo a saúde dos trabalhadores[...]";

E6 - "Promoção, prevenção, vigilância e assistência em saúde e segurança do trabalhador".

Segundo Neiverth et al. (2016); e Brasil (2012); ao CEREST cabe ainda, ações direcionadas a promoção, vigilância e assistência a saúde do trabalhador, bem como o papel de desempenhar ações de educação permanente.

\section{REALIZAÇÃO DAS AÇÕES DESENVOLVIDAS PELO CEREST}

As ações desenvolvidas pelo CEREST são realizadas através de denúncias do Ministério Público do Trabalho (MPT) e de capacitações e treinamentos da rede de saúde.

\begin{tabular}{l|l} 
Quadro no3 - Realização das ações desenvolvidas pelo CEREST \\
\hline Realizações das ações desenvolvidas pelo CEREST & Entrevistado (E1 a E4) \\
\hline $\begin{array}{l}\text { Fiscalizações e vigilância em saúde do trabalhador através de } \\
\text { denúncias do MPT, anônimas, por telefone, sindicatos, mídia, }\end{array}$ & 03 (E2, E3, e E4) \\
redes sócias etc. & 02 (E1 e E4). \\
\hline $\begin{array}{l}\text { Promoção e prevenção em saúde do trabalhador através de } \\
\text { capacitações e treinamentos }\end{array}$ & \\
\hline
\end{tabular}

As fiscalizações realizadas pela equipe multiprofissional do CEREST acontecem a partir do recebimento de denúncias provenientes principalmente do Ministério Público do Trabalho, dos sindicatos e redes sociais, denúncias via telefone, anônimas e demanda espontânea.

\footnotetext{
E1- "Após denúncias, os ficais realizam atividades de fiscalização ás empresas, na medida do possível"

E 2 e 3- "As fiscalizações são realizadas através de denúncias do MPT (Ministério Público do Trabalho), quando há uma solicitação deste para cerificar alguma situação em saúde do trabalhador; também por meio da mídia, redes sócias, sindicatos e demanda espontânea. A gente vai lá para verificar a veracidade da denúncia, embasado nas NR (Normas Regulamentadoras do Ministério do Trabalho)."

E4 -"Através de denúncias via telefone, anônimas, através do ministério público do trabalho, podem vir de várias fontes; após essas denúncias acontecem as fiscalizações onde a gente vai na empresa e fiscaliza o ambiente de trabalho checar a questão da segurança do ambiente de trabalho, em relação as máquinas, aos EPI"
}

Bevian e Ferrazzo (2014), afirmam que de fato o CEREST recebe um grande volume de denúncias de vários setores e atores sociais, como sindicatos de trabalhadores de várias classes, ouvidorias públicas e por denúncias telefônicas; sendo que a maior parte 
dessas denúncias advém do Ministério Público do Trabalho.

O CEREST atua também na organização de capacitação para os municípios da área de abrangência segundo os profissionais E1 e E4:

E1 - "[...]. São realizadas capacitações de acordo com a demanda. São estruturados cursos, seminários e ações. Essas ações acontecem em todos os 47 municípios de abrangência".

E4 - "[...]. Com relação a promoção e prevenção a gente faz capacitações e treinamentos nos municípios da nossa área de abrangência."

Com relação as capacitações vários estudos apontam que o CEREST desenvolve a capacitação de profissionais das redes de saúde, bem como a estruturação destes serviços. (Rosa et al., 2016).

\section{CATEGORIA 3 - DIFICULDADES ENCONTRADAS PELA EQUIPE}

\section{MULTIPROFISSIONAL CEREST}

$\mathrm{Na}$ categoria 3 foram apontadas as seguintes dificuldades: falta de recurso humanos, diversidade da área de atuação das empresas e localização das empresas quanto a área de abrangência.

Quadro no 4 - Dificuldades encontradas pela equipe multiprofissional do CEREST

\begin{tabular}{l|l}
\hline Dificuldades encontradas & Entrevistado (E1 a E4) \\
\hline Falta de recursos humanos & $02(E 1$ e E4) \\
\hline Diversidade da área de atuação das empresas & $02(E 3, E 2)$ \\
\hline Localização das empresas & $01(E 2)$ \\
\hline
\end{tabular}

Os profissionais relatam que a falta de recursos humanos atualmente se dá pelo processo de reestruturação e transição do serviço, relacionada a troca de gestão pública.

E1 "No momento devido as demissões e reestruturação da equipe, há falta de funcionários".

E4 "A equipe está muito reduzida, a principal dificuldade agora é pessoas para trabalhar".

Santos, Boavista e Vilarinho (2015), referem que a redução do numero de recursos humanos por uma conjuntura atual em uma equipe de saúde tem como conseqüência um atendimento de má qualidade.

Para Brasil 2005, a equipe mínima do CEREST regional deve ser composta por 10 profissionais; 4 profissionais de nível médio, com pelo menos 2 auxiliares de enfermagem. 6 profissionais de nível universitário, sendo ao menos 2 médicos (20 horas semanais) e 1 enfermeiro (40 horas semanais).

Observou-se que atualmente, o CEREST está dividido em dois núcleos de ações: 1 voltado as capacitações e outro voltado as fiscalizações. No momento o serviço conta com 2 profissionais atuando nas fiscalizações dos ambientes de trabalho, 1 nas capacitações e 1 na coordenação geral.

O acumulo de tarefas a serem executadas pelos profissionais; foi evidenciado perante um cronograma de fiscalizações a serem realizadas em um curto período de tempo. 
Da mesma forma, as diversidades da área de atuação das empresas, também apontada como uma das dificuldades da equipe, está relacionada a formação técnica dos fiscais.

E2 “- [...] A gente fiscaliza tudo que é empresa, não interessa o que ela faz; se é pesca, confecção, plástico, sapato [...]".

E3 "- Cada caso é um caso, a gente está sempre encontrando coisas diferentes, porque cada empresa é um ramo diferente; não se sabe o que a gente vai encontrar, não se sabe que tipo de adequação a gente vai pedir para aquela empresa, para que não aconteça possíveis acidentes de trabalho, para que elas possam dar melhores condições para os funcionários. Então nosso trabalho é bem diversificado [...]".

Em qualquer das opções é preciso ampliar o quadro de AFT dedicados a SST, oferecendoIhes qualificação em áreas afins, como Higiene e Segurança do Trabalho e Saúde do Trabalhador, assim como assegurar que as ações de SST tenham a amplitude técnica necessária para coibir e controlar a exposição dos trabalhadores a situações de risco e, em especial, evitar as mortes e lesões incapacitantes, em sua grande maioria evitáveis (VASCONCELOS, 2014, p. 98).

E se tratando de formação técnica dos agentes fiscalizadores, o processo deverá ser feito de forma distinta conforme os níveis de formação de cada profissional, respeitando não somente suas capacidades técnicas, mas também as tecnológicas relacionadas a cada área de atuação do CEREST (VASCONCELLOS; GOMEZ; MACHADO, 2014).

E2 "- Uma das maiores dificuldades é a localização das empresas"

A localização e área da abrangência das empresas, apontadas como sendo uma das dificuldades da equipe; se deve ao número de cidades que o CEREST abrange, que vai de Passo de Torres e Paulo Lopes; totalizando 47 municípios abrangidos. Também existe a dificuldade de chegar a determinadas empresas para fiscalizar, pois o acesso é ruim devido à falta infraestrutura como ruas sem calçamentos e empresas sem placas de identificação.

\section{CATEGORIA 4 -ATENDIMENTOS ASSISTENCIAIS PELO CEREST.}

Quadro no5 - Atendimentos assistenciais pelo CEREST

\begin{tabular}{l|l}
\hline Atendimentos assistenciais pelo CEREST & Entrevistado (E1 a E4) \\
\hline Não & 03 (E1, E2 e E3) \\
\hline Sim & $01(E 4)$ \\
\hline
\end{tabular}

Três das entrevistadas responderam que não há atendimento assistencial no CEREST. Essas expressões ficam claras nas falas a seguir:

E1- "O CEREST não faz atendimentos assistenciais. $O$ atendimento é feito pelo ambulatório de saúde do trabalhador".

E2 e 3 - "Não é o CEREST que presta serviços de assistência, é o ambulatório de saúde do trabalhador que fica aqui no mesmo prédio, mas não é do CEREST".

Uma das entrevistadas, portanto, respondeu que sim, que o CERET presta serviços assistências em saúde do trabalhador; sendo que a assistência está relacionada ao receber 
essa pessoa e encaminhá-la ao ambulatório de saúde do trabalhador que fica ao lado, ocupando o mesmo espaço físico onde está situado o CEREST.

E 4 -"Sim. O trabalhador que tiver qualquer problema de saúde relacionado ao trabalho ele é encaminhado para cá, para o ambulatório de saúde do trabalhador; pode vir através da atenção básica, através de sindicatos; ele vai ser atendido pelo médico do trabalho que vai fazer um nexo causal. Esse é o objetivo da assistência, estabelecer o nexo, ele não vai ser tratado aqui".

O Cerest segundo IMBRIZI; KEPPLER e VILHANUEVA (2013), é um serviço que oferece ações de promoção, vigilância e assistência à saúde do trabalhador.

Assistência: atendimento individualizado aos trabalhadores que demandam os serviços do CEREST, através da equipe multiprofissional de acordo com a necessidade do caso, cuja atuação se dará no sentido de estabelecer o nexo causal entre os processos/ambientes de trabalho e a saúde do trabalhador (CEREST, 2017).

Realização de atendimento aos trabalhadores acometidos por doenças e acidentes de trabalho, buscando diagnósticos de tratamentos específicos e o estabelecimento do nexo causal. (CEREST, 2017).

Vários estudos nos mostram que os atendimentos assistenciais a saúde do trabalhador é ofertada pelos diversos serviços que compõem a rede sentinela. Segundo as falas e mediante observações, notou-se que o serviço de assistência não é feito pelo CEREST e sim pelo ambulatório de saúde do trabalhador que faz parte da RENAST.

\section{CATEGORIA 5 - PROCURA POR ATENDIMENTOS ASSISTENCIAIS NO CEREST.}

Quadro n6 - Procura por atendimentos assistenciais no CEREST

\begin{tabular}{l|l}
\hline Procura por atendimentos assistenciais no CEREST & Entrevistado (E1 a E4) \\
\hline Sim & $\mathbf{0 4}(\mathrm{E} 1, \mathrm{E} 2, \mathrm{E3}$ e E4) \\
\hline Não & $\mathbf{0}$ \\
\hline
\end{tabular}

Todas as entrevistadas relataram que há procura por atendimentos assistenciais no CEREST. Essa procura ocorre pelo fato de o CEREST e ambulatório funcionarem na mesma estrutura física.

E1" - Sim, são encaminhados para o ambulatório de outros serviços da rede".

E2 e 3 "É agendado tudo ali; no ambulatório".

E4 "- Existe sim, e a gente orienta a forma que tem que ser feito o encaminhamento, porque as vezes vem pessoas que não tem nada a ver com saúde do trabalhador que acha que a gente faz consulta, e não é isso".

Durante a pesquisa, pode-se observar que existe de fato essa procura por parte dos trabalhadores de várias empresas e locais de trabalho, que desconhecem o fluxo de atendimento do serviço. Esses usuários são orientados quanto ao atendimento ao chegarem no local. 


\section{CATEGORIA 6 - ATENDIMENTO ASSISTENCIAL COMO AÇÃO DO CEREST.}

Quadro no7 - Atendimento assistencial como ação do CEREST

\begin{tabular}{l|l}
\hline Atendimento Assistencial como ação do CEREST & Entrevistado (E1 a E4) \\
\hline Não é o papel do CEREST & 02 (E2 e E3) \\
\hline É um trabalho complementar & $01(E 4)$ \\
\hline Inviável pela forma que o serviço está estruturado & $01(E 1)$ \\
\hline
\end{tabular}

Nesta categoria duas das entrevistadas colocam claramente que o atendimento assistencial não é papel do CEREST. Uma relata o atendimento assistencial como um trabalho complementar ao do CEREST. Outra diz que é inviável pela forma que o serviço está estruturado.

Não a clareza das ações do CEREST para a população de forma geral e para os serviços de atenção primaria em saúde.

E1 "- Pelo pouco tempo que atuo neste serviço, acho inviável, pelo menos da forma como o serviço esta estruturado".

E 2 e 3 "- Não é papel do CEREST fazer assistência, o que as vezes acontece é de as pessoas chegarem para nós enquanto fiscal e perguntar, aí a gente orienta. Eles perguntam: sofri um acidente de trabalho e a empresa não me deu condições nenhuma, não me orientou, não emitiu uma CAT, o que eu faço? Como procedo? Assim a gente só orienta o ambulatório";

E4 "- Esse atendimento assistencial é um trabalho complementar e ele faz programa da RENAST, está incluso na vigilância em saúde do trabalhador. A rede precisa estar mais articulada com as outras vigilâncias em saúde, precisa estar mais integrado".

Pode-se observar que há uma divergência de conhecimento sobre as atribuições do CEREST; não só para os serviços adjacentes, mas também dentro da própria instituição.

\section{CONCLUSÃo}

Com esta pesquisa, constatou-se que existem dificuldades de vigilância em saúde do trabalhador enfrentadas pela equipe que atua no CEREST.

No momento a equipe encontra-se reduzida, sendo este um dos principais problemas apontados no estudo. Observou-se que essa redução de recursos humanos se deu pela atual conjuntura política de troca de gestão dos serviços de saúde, inclusive do CEREST. Em decorrência desta realidade, os profissionais têm desenvolvido suas ações voltadas mais para o processo de fiscalização do ambiente e das condições de trabalho; que chegam através do MTE, e que tem data para serem realizadas. Assim a atual administração vem tentando estruturar o serviço com os recursos mais viáveis na espera que os gestores lhe proporcione os recursos humanos necessários para o serviço.

Em contra partida e sem entender toda essa mudança estão o trabalhadores que são o foco do serviço mas ficam de certa forma desassistidos pelo serviço que tem tentado manter-se mesmo com a demanda crescente dia após dia em relação ao número de funcionários que compõem a equipe. 
As demais dificuldades vem alencadas a falta de recursos humanos pois se torna ainda mais difícil fazer as fiscalizações se tratando de um abrangência ampla e o contato com todos os municípios para dar suporte ao serviço também fia defasado.

Entende-se que esta pesquisa tem uma importância acadêmica e de certa forma sócio- política pois pode ser usada como instrumento concreto para consolidação formal e adesão junto ao gestores para conseguir novos recursos melhorando assim não só o CEREST mais toda saúde do trabalhador.

\section{REFERÊNCIAS}

BRASIL. Constituição da República Federativa do Brasil. Brasília, DF: Senado, 1988.

, Lei no 8080/90, de 19 de setembro de 1990. Brasília, 1990.

, Portaria MS no 1.679/GM, de 19 de setembro de 2002. Brasília, 2002.

Portaria MS no 2.728/GM, de 11 de novembro de 2009. Brasília, 2009.

Rede Nacional de Atenção Integral à Saúde do Trabalhador: Manual de Gestão e Gerenciamento. São Paulo: Hemeroteca Sindical Brasileira, 2006.

\section{PORTARIA № 2.437, DE 7 DE DEZEMBRO DE 2005}

CARDOSO, Mariana de Castro Brandão; ARAUJO, Tânia Maria de. Os Centros de Referências em Saúde do Trabalhador e as ações em saúde mental: um inquérito no Brasil. Rev. bras. saúde ocup. São Paulo, v. 41, e7, 2016. Available from <http://www.scielo.br/scielo.php?script=sci_arttext\&pid=S030376572016000100203\&lng=en\&nrm=iso >. access on 02 Nov. 2016. Epub Aug 29, 2016. http://dx.doi.org/10.1590/2317-6369000118115.

CEREST. Secretaria do Estado de Saúde do Piauí. Centro Estadual de Referência em Saúde do Trabalhador, 2017. Disponível em: < http://www.saude.pi.gov.br/divisa/cerest> Acesso em: 12 jun. 2017.

CEREST. Secretaria Municipal de Saúde de ljuí - RS. Centro Estadual de Referência em Saúde do Trabalhador, 2017. Disponível em: < http://www.cerestijui.com/publicacao-1351acoes.fire> Acesso em: 12 jun. 2017

DALDON, Maria Teresa Bruni; LANCMAN, Selma. Vigilância em Saúde do Trabalhador: rumos e incertezas. Rev. bras. saúde ocup. São Paulo, v. 38, n. 127, p. 92-106, June 2013. Available from <http://www.scielo.br/scielo.php?script=sci_arttext\&pid=S0303$76572013000100012 \& \operatorname{lng}=$ en\&nrm=iso $>$. access on 02 Nov. 2016. http://dx.doi.org/10.1590/S0303-76572013000100012.

DIAS, Elizabeth Costa; SILVA, Thais Lacerda e. Contribuições da Atenção Primária em Saúde para a implementação da Política Nacional de Saúde e Segurança no Trabalho (PNSST). Rev. bras. saúde ocup. São Paulo, v. 38, n. 127, p. 31-43, June 2013. Available from <http://www.scielo.br/scielo.php?script=sci_arttext\&pid=S0303$76572013000100007 \&$ lng $=$ en\&nrm=iso $>$. access 
on 02 Nov. 2016. http://dx.doi.org/10.1590/S0303-76572013000100007.

DURAN, Erika Christiane Marocco; ROBAZZI, Maria Lucia do Carmo Cruz; MARZIALE, Maria Helena Palucci. Conhecimento de enfermagem em saúde do trabalhador oriundo de dissertações e teses. Revista Gaúcha de Enfermagem, Porto Alegre, Rs, v. 3, n. 28, p.416-423, 2007.

FERNANDES, Almesinda Martins de O.; GUIMARÃES, Zileny da Silva. Saúde-Doença do Trabalhador: Um guia para os profissionais. Gioânia: Ab, 2007. 296 p. (Coleção Saúde e segurança do Trabalhador).

GALDINO, Adriana; SANTANA, Vilma Sousa; FERRITE, Silvia. Os Centros de Referência em Saúde do Trabalhador e a notificação de acidentes de trabalho no Brasil. Cad. Saúde Pública, Rio de Janeiro, v. 28, n. 1, p. 145-159, Jan. 2012 . Available from $<$ http://www.scielo.br/scielo.php?script=sci_arttext\&pid=S0102-

$311 \times 2012000100015 \&$ Ing $=$ en\&nrm $=$ iso $>$. access

on 02 Nov. 2016. http://dx.doi.org/10.1590/S0102-311X2012000100015.

GERHARDT, Tatiana Engel; SILVEIRA, Denise Tofo (Org.). Métodos de pesquisa. Porto Alegre: Ufrgs, 2009. 120 p. (Educação a Distância).

IBAñES, Rachel Ramos. Gestão de pessoas por competências: os desafios enfrentados no serviço público. Conteudo Juridico, Brasilia-DF: 11 mar. 2016. Disponivel em:

<http://www.conteudojuridico.com.br/?artigos\&ver=2.55411\&hl=no>. Acesso em: 27 maio 2017.

IMBRIZI, Jaquelina Maria; KEPPLER, Isabel Lopes do Santos; VILHANUEVA, Marcelo Soares. Grupo dos Novos: relato de uma experiência de estágio com grupos de acolhimento de trabalhadores em um Centro de Referência em Saúde do Trabalhador (Cerest). Rev. bras. saúde ocup. São Paulo, v. 38, n. 128, p. 302-314, Dec. 2013

Available from <http://www.scielo.br/scielo.php?script=sci_arttext\&pid=S0303$76572013000200017 \&$ Ing $=$ en\&nrm=iso $>$. access on 02 Nov. 2016. http://dx.doi.org/10.1590/S0303-76572013000200017.

JACQUES, Camila Corrêa; MILANEZ, Bruno; MATTOS, Rita de Cássia Oliveira da Costa. Indicadores para Centros de Referência em Saúde do Trabalhador: proposição de um sistema de acompanhamento de serviços de saúde. Ciênc. saúde coletiva, Rio de Janeiro, v. 17, n. 2, p. 369-378, Feb. 2012 . Available from $<$ http://www.scielo.br/scielo.php?script=sci_arttext\&pid=S1413$81232012000200011 \&$ Ing $=$ en\&nrm=iso $>$. access on 02 Nov. 2016. http://dx.doi.org/10.1590/S1413-81232012000200011.

KANAANE, Roberto. Comportamento Humano nas Organizações: O Homem Rumo ao Século XXI. 2. ed. São Paulo: Atlas, 1999.

LACAZ, F. A. C. Saúde do Trabalhador: um estudo sobre as formações discursivas da academia, dos serviços e do movimento sindical. Tese em saúde coletiva. Campinas: Faculdade de Ciências Médicas da Universidade Estadual de Campinas - Unicamp, 1996.

LACAZ, Francisco Antonio de Castro; FLORIO, Solange Maria Ribeiro. Controle social, mundo do trabalho e as Conferências Nacionais de Saúde da virada do século XX.Ciênc. saúde coletiva, Rio de Janeiro, v. 14, n. 6, p. 2123-2134, Dec. 2009. Available from 
<http://www.scielo.br/scielo.php?script=sci_arttext\&pid=S1413-

$81232009000600019 \&$ Ing $=$ en \&nrm=iso $>$. access

on 02 Nov. 2016. http://dx.doi.org/10.1590/S1413-81232009000600019.

LEAO, Luís Henrique da Costa; CASTRO, Alexandre de Carvalho. Políticas públicas de saúde do trabalhador: análise da implantação de dispositivos de institucionalização em uma cidade brasileira. Ciênc. saúde coletiva, Rio de Janeiro, v. 18, n. 3, p. 769-

778, Mar. 2013. Available from

<http://www.scielosp.org/scielo.php?script=sci_arttext\&pid=S1413-

$81232013000800023 \&$ lng $=$ en \&nrm=iso $>$. access

on 02 Nov. 2016. http://dx.doi.org/10.1590/S1413-81232013000800023.

MACHADO, Jorge Mesquita Huet et al. Situação da Rede Nacional de Atenção Integral em Saúde do Trabalhador (Renast) no Brasil, 2008-2009. Rev. bras. saúde ocup. São Paulo, v. 38, n. 128, p. 243-256, Dec. 2013. Available from $<$ http://www.scielo.br/scielo.php?script=sci_arttext\&pid=S0303-

$76572013000200012 \&$ lng $=$ en \&nrm=iso $>$. access

on 02 Nov. 2016. http://dx.doi.org/10.1590/S0303-76572013000200012

MINAYO, Maria Cecília de Souza. O Desafio do Conhecimento: Pesquisa Qualitativa em Saúde. 11. ed. São Paulo: Hucitec, 2008. 393 p.

RIBEIRO, Fátima Sueli Neto. Vigilância em Saúde do Trabalhador: a tentação de engendrar respostas às perguntas caladas. Rev. bras. saúde ocup. São Paulo, v. 38, n . 128, p. 268-279, Dec. 2013. Available from

<http://www.scielo.br/scielo.php?script=sci_arttext\&pid=S0303-

$76572013000200014 \&$ lng $=$ en\&nrm=iso $>$. access

on 02 Nov. 2016. http://dx.doi.org/10.1590/S0303-76572013000200014.

SANTOS, Ana Paula Lopes dos; LACAZ, Francisco Antonio de Castro. Apoio matricial em saúde do trabalhador: tecendo redes na atenção básica do SUS, o caso de Amparo/ SP. Ciênc. saúde coletiva, Rio de Janeiro, v. 17, n. 5, p. 1143-1150, May 2012. Available from <http://www.scielo.br/scielo.php?script=sci_arttext\&pid=S1413-

$81232012000500008 \& \operatorname{lng}=\mathrm{en} \& \mathrm{nrm}=\mathrm{iso}>$. access

on 02 Nov. 2016. http://dx.doi.org/10.1590/S1413-81232012000500008.

SANNA, Maria Cristina. Os processos de trabalho em Enfermagem. Revista Brasileira de Enfermagem, Brasilia, p.221-224, 2007.

SOUZA, Thiago Santos de; VIRGENS, Liliam Silva das. Saúde do trabalhador na Atenção Básica: interfaces e desafios. Rev. bras. saúde ocup. São Paulo, v. 38, n. 128, p. 292301, Dec. 2013 . Available from <http://www.scielo.br/scielo.php?script=sci_arttext\&pid=S0303-

$76572013000200016 \&$ lng $=$ en $\&$ nrm $=$ iso $>$. access

on 02 Nov. 2016. http://dx.doi.org/10.1590/S0303-76572013000200016.

TORRES, Amélia Romana Almeida et al. O ADOECIMENTO NO TRABALHO:

Repercussões na vida do trabalhador e de sua família. Sanare, Sobral, v. 10, n. 1, p.4248, 2011.

VASCONCELOS, Fernando Donato. Atuação do Ministério do Trabalho na fiscalização das condições de segurança e saúde dos trabalhadores, Brasil, 1996-2012. Rev. bras. 
saúde ocup., São Paulo, v. 39, n. 129, p. 86-100, June 2014 . Available from <http://www.scielo.br/scielo.php?script=sci_arttext\&pid=S0303$76572014000100086 \&$ Ing=en\&nrm=iso $>$. access on 30 May 2017. http://dx.doi.org/10.1590/0303-7657000072012.

VASCONCELLOS, Luiz Carlos Fadel de; ALMEIDA, Carmen Verônica Barbosa de; GUEDES, Dimitri Taurino. Vigilância em saúde do trabalhador: passos para uma pedagogia. Trab. educ. saúde, Rio de Janeiro, v. 7, n. 3, p. 445-462, Nov. 2009 . Available from <http://www.scielo.br/scielo.php?script=sci_arttext\&pid=S1981 $77462009000300004 \&$ lng $=e n \& n r m=i s o>$. access on 02 Nov. 2016. http://dx.doi.org/10.1590/S1981-77462009000300004.

; GOMEZ, Carlos Minayo; MACHADO, Jorge Mesquita Huet. The gap between what has been defined and what is still pending in occupational health surveillance. Ciênc. saúde coletiva, Rio de Janeiro, v. 19, n. 12, p. 4617-4626, Dec. 2014 . Available from <http://www.scielosp.org/scielo.php?script=sci_arttext\&pid=S1413$81232014001204617 \& \operatorname{lng}=$ en $\&$ nrm=iso $>$. access on 02 Nov. 2016. http://dx.doi.org/10.1590/1413-812320141912.13602014.

QUILIAO, Paula Lamb; FASSA, Anaclaudia Gastal; RESTREPO, María Clara. Processo de implantação de um Centro Regional de Referência em Saúde do Trabalhador no Rio Grande do Sul. Rev. bras. saúde ocup. São Paulo, v. 38, n. 128, p. 257-267, Dec. 2013 . Available from <http://www.scielo.br/scielo.php?script=sci_arttext\&pid=S0303$76572013000200013 \&$ Ing $=e n \& n r m=i s 0>$. access on 02 Nov. 2016. http://dx.doi.org/10.1590/S0303-76572013000200013. 